\title{
Verein zur Förderung der klinischen Verhaltenstherapie
} (VFKV)

Mitteilungen des Vorstands:

Am 1. Oktober 1991 hat ein neuer Kurs begonnen.

Da ständig neue Aufgaben anfallen, haben wir begonnen, die Arbeit zu dezentralisieren und feste Arbeitsbereiche im Ausbildungsausschuß zu verteilen.

Zuständig für die medizinische Ausbildung sind:

Dr. Tröstl, Dr. Utz, Dr. Zaudig

Zuständig für das Gruppen-Curriculum sind:

Dr. Utz, Dr. Köhler

Vertreter des Instituts im Ausschuß der kooperierenden Institute ist Herr Hauke.

Weitere Arbeitsbereiche sollen besetzt werden.

Der Vorstand begrüßt das sich abzeichnende Zusammengehen des FKV und der DAVT. Eine gemeinsame Organisation, insbesondere der beiden Verbänden angeschlossenen AusbildungsInstitute mit einheitlicher Geschäftsführung halten wir für wünschenswert.

Zusammen mit der Privaten Bayerischen Akademie für Psychotherapie, dem Klinikum rechts der Isar und dem Max-Planck-Institut für Psychiatrie führte der VFKV eine Arbeitstagung zu dem Thema «Verhaltenstherapie im Alter» durch. Aufgrund des regen Zuspruchs und der positiven Rückmeldungen wurde beschlossen, im nächsten Herbst eine weiterführende Veranstaltung anzubieten.

Kontaktadresse: VFKV Heßstraße 90 W-8000 München 40 Tel. 089/186605

Mitteilungen der Verbände 